\title{
Truenat - A Novel Diagnostic Tool for Rapid Detection of Mycobacterium tuberculosis and Rifampicin Resistance in Pulmonary Samples
}

\author{
J. Vijayalakshmi, A. Surekha, A. Renuka Devi and S. Uma Devi* \\ Department of Microbiology, Kurnool Medical College, Kurnool, AP. 518002, India \\ *Corresponding author
}

\section{A B S T R A C T}

\section{Keywords}

Chip based assay,

Trueprep AUTO

Universal Cartridge

Based Sample Prep

Device TruenatTM

MTB chip

\section{Article Info}

Accepted:

10 September 2019

Available Online:

10 October 2019
Tuberculosis is a major public health problem and second largest cause of death among infectious diseases. Rapid diagnosis of tuberculosis and detection of drug resistance is essential for effective disease control. Control of TB is hindered by the lack of a simple and effective diagnostic tool that can be utilised in resource limited settings. Molecular techniques using Real time PCR technology have gained momentum for rapid diagnosis and detection of drug resistance. To study the prevalence of Pulmonary Tuberculosis and Rifampicin resistance in Kurnool district by using Truenat chip based micro PCR system and to understand the demographical distribution of cases attending the tertiary care hospital. This was a prospective study done during the period from March 2019 to Aug 2019. All the presumptive TB cases with chest symptoms attending Govt.General Hospital, Kurnool were included in the study. Sputum samples were processed by Truenat, a chip based nucleic acid amplification test (Molbio diagnostics pvt. Itd., Goa). The procedure included two steps: first- sputum samples were processed for separation of DNA by using True prep device. Second-Presence of MTB was detected by Truelab ${ }^{\text {TM }}$ Real Time micro PCR Analyzer. All the MTB positive samples were tested for the presence of Rifampicin resistance by using another specialized chip. A total of 1825 sputum samples were processed, out of which $368(20.16 \%)$ were positive for MTB. Rifampicin resistance was detected in $70(19.02 \%)$. Positivity was more in males $63.23 \%$ as compared to females $36.76 \%$. Majority of the cases were in the age group 40-60 years (46.58\%), followed by $20-40$ years $(33.04 \%)$. Truenat is a simple method and laboratory technician with minimal training can perform the procedure. The Turn Around Time (TAT) is approximately one hour. As no special infrastructure or Airconditioning system is required to set up the device it can be used in resource limited settings for rapid diagnosis of MTB and detection of Rifampicin resistance.

\section{Introduction}

Tuberculosis causes the highest number of deaths globally despite the availability of potent anti-TB drugs.
The global burden of TB remains enormous. As per Global TB report 2017, more than 9 million new tuberculosis cases and 1.6 million deaths occur annually worldwide. Estimated MDR/RR cases are 6 lakhs. India is the highest TB burden country. 1/4th of the global 
annual new cases occur in India. Incidence is 2.7 million cases annually and mortality is 4.35 lakhs per year (1). Over $25 \%$ of patients seeking care in India's public sector are neither diagnosed nor started on treatment (2). Tuberculosis (TB) is the second largest killer worldwide, after HIV and is the leading cause of death in HIV patients. Pulmonary TB spreads through aerosols and is highly contagious. Over $80 \%$ of TB infections are pulmonary and if left untreated, a pulmonary TB patient can infect up to 10-15 other people through close contact over the course of a year(3). Due to the highly infectious nature of pulmonary TB, it is important to diagnose and treat the disease very early. Despite the availability of highly effective treatment for decades, TB remains a major global health problem mainly because of poor case detection(4). The most common method for diagnosing pulmonary TB worldwide is sputum smear microscopy. However sensitivity of direct smear microscopy is low and estimates range from $30 \%$ to $70 \%$. It is even lower in case of HIV-infected patients. Culture is more sensitive than microscopy and is considered the current gold standard. Culture requires specialized and controlled laboratory facility and highly skilled manpower and takes 2 to 6 weeks to provide the result. Automated methods and Molecular techniques such as polymerase chain reaction (PCR) or Real Time PCR are much more sensitive than microscopy and culture. However these tests have so far been restricted to centralized reference laboratories as they require skilled manpower and elaborate infrastructure. Also the turnaround time for results could take a few day $(5,6)$.

Moreover Drug resistance is a major issue in the treatment of tuberculosis. Drug resistance is because of either mismanagement of TB patients- wrong diagnosis, delay in diagnosis, wrong or interrupted treatment and injudicious use of both first and second line drugs. Multiple approaches to improve diagnosis of
TB are in development. Amongst these are CBNAAT (GeneXpert) and LPA, endorsed by WHO to be used in RNTCP for rapid diagnosis of MTB and detection of Rifampicin resistance (7). These require uninterrupted power supply, air conditioning system, proper infrastructure and trained personnel. Patients have to travel to nearest testing centre or samples need to be transported. This limits its use in peripheral settings and Active Case Finding program (8).

The need is for accurate, feasible, rapid, affordable, and if possible, near-point-of-case TB diagnostic tests for use in resource limited settings $(9,10)$. The Truelab ${ }^{\text {TM }}$ Real Time micro PCR System enables decentralization and near patient diagnosis of MTB by making real time PCR technology rapid, simple, robust and user friendly and offering "sample to result" capability even at resource limited settings. It is supplied by Molbio diagnostics pvt. ltd., Goa, funded by Bigtec labs, India. This test has been evaluated by the premiere institutes for its sensitivity and specificity and has been recommended in health care settings $(9,11)$. It is extensively validated and licensed by Indian FDA. It has sensitivity of $99 \%$ and specificity of $100 \%$. The MTB strain H37Rv from Zeptometrix was used for LOD (Limit of Detection) determination. LOD was determined to be $100 \mathrm{CFU} / \mathrm{ml}$ in sputum sample.

To know the prevalence of Tuberculosis in Kurnool district by using chip based Truelab $^{\text {TM }}$ Real Time micro PCR System

To understand the demographics of TB

To know the prevalence of Rifampicin resistance among New and Previously treated cases.

\section{Materials and Methods}

Sputum samples of all the Presumptive pulmonary TB cases were collected in universal container. Having supplied by the 
Govt to our institute in March 2019, the Truenat device was used for this study.

Each sample was processed by Trueprep Auto MTB sample pretreatment pack which employs a combination of reagents. These reagents homogenise the sample to release the bacilli, concentrate to get better yields of bacilli and also discard potentially inhibitory substances. The pretreated sample is loaded in Trueprep AUTO Universal Cartridge Based Sample Prep kit (Figure 1) and Device (Figure 2) to enable further extraction and purification of the bacterial DNA so that it is free from PCR inhibitors. It is a light weight, portable device designed to operate at room temperature. It operates on mains and/or rechargeable battery and is fully automatic with minimal hands on time. The cartridge based extraction process is quick, reliable and efficient and does not require highly skilled personnel to carry out the extraction process. All the waste from processing of the sample is contained within the cartridge dump area thus posing no risk from potentially biohazardous material. At the end of processing the bound DNA is eluted and collected in elution chamber. The entire process takes $20 \mathrm{~min}$. The elute is transferred to the Elute Collection tube (ECT).

The Truenat ${ }^{\mathrm{TM}}$ MTB chip(Figure 4) is placed on the chip tray of the Truelab ${ }^{\mathrm{TM}}$ Real Time micro PCR Analyzer (Figure 3). Six $\mu \mathrm{L}$ of the purified DNA is then dispensed using the provided micropipette and tip into the microtube containing freeze dried PCR reagents and allowed to stand for 30-60 seconds to get a clear solution. Six $\mu \mathrm{L}$ of this clear solution is then pipetted out and dispensed into the reaction well of the Truenat $^{\mathrm{TM}}$ MTB chip and the test is started. A positive amplification causes the dual labelled fluorescent probe to release the fluorophore in an exponential manner which is then captured by the built-in opto-electronic sensor and displayed as amplification curve on the analyzer screen, on a real time basis during the test run. The Cycle threshold $(\mathrm{Ct})$ is defined as the number of amplification cycles required for the fluorescent signal to cross the threshold. In the case of negative samples, amplification does not occur and a horizontal amplification curve is displayed on the screen during the test run.

At the end of the test run, a MTB "DETECTED" or "NOT DETECTED" result is displayed and in positive cases, quantitative value is also displayed on the screen. Based on the $\mathrm{Ct}$ of the internal positive control (IPC), the validity of the test run is also displayed. The IPC is a full process control that undergoes all the processes the specimen undergoes - from extraction to amplification thereby validating the test run from sample to result.

The target sequence for this kit is part of the ribonucleoside-diphosphate reductase gene, the product of which provides the precursor for DNA synthesis. The region selected is specific to the MTB complex. The used Truenat $^{\mathrm{TM}}$ MTB micro PCR chip in submerged in freshly prepared $0.5 \%$ sodium hypochlorite solution for 30 minutes before disposal as per the standard medical waste disposal guidelines.

The samples which were positive for MTB were loaded into another chip designed for detection of rifampicin resistance (Truenat $^{\mathrm{TM}}$ MTB/RIF micro PCR chip) and loaded into Truelab $^{\mathrm{TM}}$ Real Time micro PCR Analyzer.

\section{Results and Discussion}

A total of 1825 sputum samples were processed during the period from March 2019 to Aug 2019. Out of these 368 (20.16\%) were positive for MTB and 1457 (79.83\%) were negative for MTB.

Out of 368 MTB positive cases, Rifampicin 
resistance was detected in $70(19.02 \%)$ and 298 (80.97\%) cases were Rifampicin sensitive. Most of the samples were from age group 40-60 (46.58\%) followed by 20-40 (33.04\%). Out of the total 1825 cases, Males were 1154 (63.23\%); amongst them, MTB was detected in 274 (23.74\%) and Rifampicin resistance seen in $53(19.34 \%)$. Females contributed to 671 (36.76\%); amongst them MTB was detected in $94(14.01 \%)$ and Rifampicin resistance seen in $17(18.08 \%)$.

Out of the total 1825 cases, New cases were 1260; amongst these MTB was detected in $206(16.34 \%)$ and Rifampicin resistance in 28 (13.59\%). Previously treated cases were 565; amongst these MTB was detected in 162 (28.67\%) and Rifampicin resistance in 42 (25.92\%). A major WHO priority for TB diagnostics is to implement a rapid, sputumbased molecular test to replace smear microscopy at the peripheral level (i.e., designated microscopy centres and attached primary healthcare facilities) $(9,11,12)$. RNTCP has recently developed National Strategic Plan (NSP) to work towards achieving the goal of eliminating $\mathrm{TB}$ by 2025(12). Drug resistant TB (DRTB) is a major impediment to achieve this goal (13). As per the Drug resistance survey conducted in India in 2014-16, the prevalence of MRDTB was $2.84 \%$ and $11.6 \%$ among new and previously treated TB cases respectively (2).
Best method to control TB is early diagnosis and initiation of appropriate treatment. Truenat Real time quantitative micro PCR assay serves the need of the day as it is a rapid, portable and can be easily used in resource limited settings. It reduces the logistical hurdles involved with transportation of sputum samples to distant referral laboratories (12). The Turn around time is about one hour which allows same day diagnosis, same day treatment decision and initiation (13). A limitation of Truenat is, it cannot detect MDRTB and resistance to second line injectables which is of most significance in high burden countries like India. But as compared to any other molecular method, it is very effective(11). In the present study, out of the total 1825 sputum samples, Mycobacterium tuberculosis was detected in 368 samples $(20.16 \%)$. Rifampicin resistance was seen in $70(19.02 \%)$. People in the age group of 40-60 were in maximum numbers (46.58\%). Males were predominantly affected (63.23\%). Rifampicin resistance was more in males (19.34\%) and among previously treated cases $25.92 \%$. Similar results were observed in many studies (14) (Table 1)

In order for wide availability as well as affordability even in peripheral settings, there is a need for make in India diagnostics to cover millions of people under risk of tuberculosis (Fig. 5 and 6).

Table.1 Age wise distribution of samples

\begin{tabular}{|c|c|}
\hline Age in years & No. of samples \\
\hline $\mathbf{0 - 2 0}$ & $116(06.35 \%)$ \\
\hline $\mathbf{2 0 - 4 0}$ & $603(33.04 \%)$ \\
\hline $\mathbf{4 0 - 6 0}$ & $850(46.58 \%)$ \\
\hline$>\mathbf{6 0}$ & $256(14.02 \%)$ \\
\hline Total & 1825 \\
\hline
\end{tabular}


Fig.1 Trueprep AUTO Universal Cartridge Based Sample Prep kit

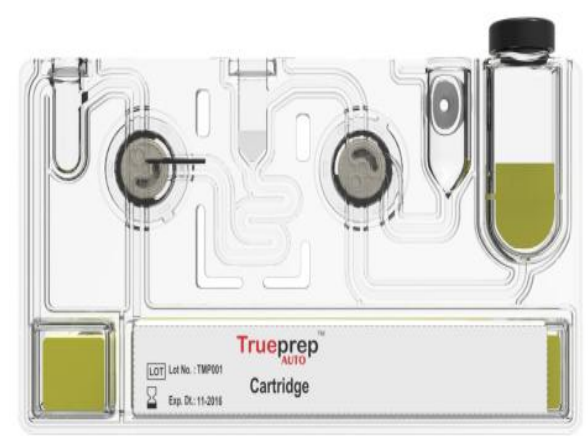

Fig.2 Trueprep AUTO Universal Cartridge Based Sample Prep device



Fig.3 Truelab ${ }^{\mathrm{TM}}$ Real Time micro PCR Analyzer

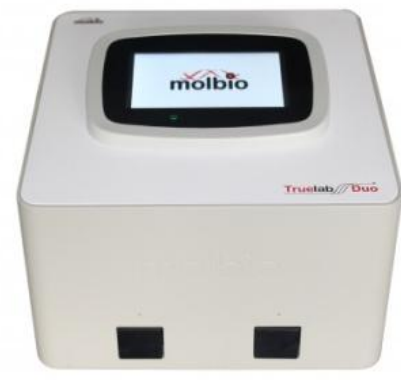

Fig.4 Truenat ${ }^{\mathrm{TM}}$ MTB chip

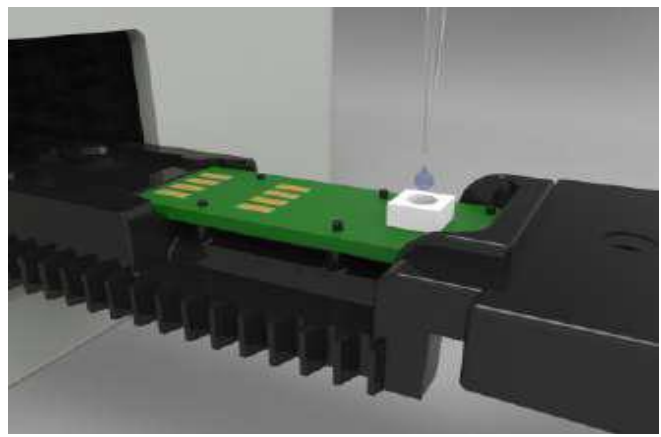


Fig.5

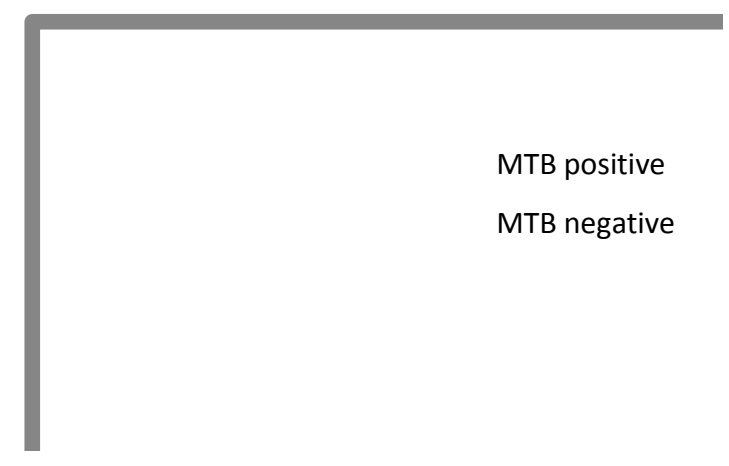

Fig.6

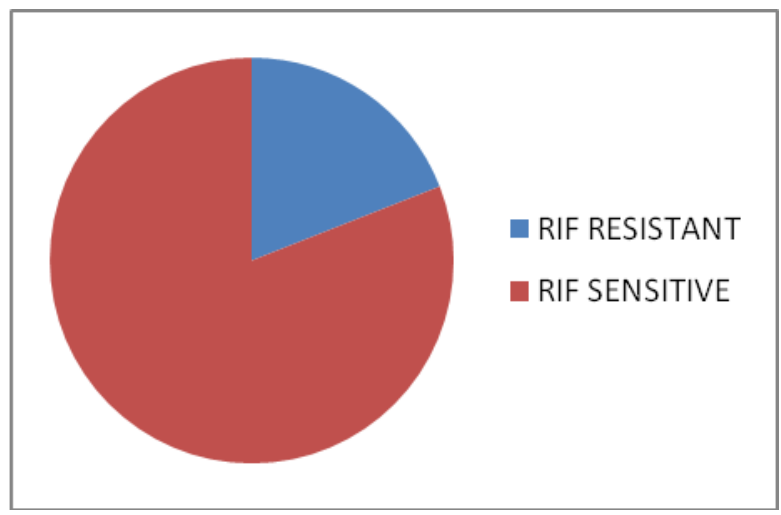

Chart.1 Results obtained gender wise

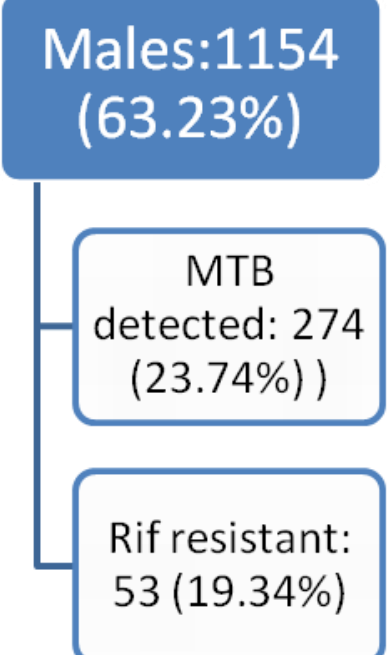

$$
\begin{aligned}
& \text { Females: } 671 \\
& \begin{array}{c}
(36.76 \%) \\
\begin{array}{c}
\text { MTB } \\
\text { detected: } 94 \\
(14.01 \%)
\end{array} \\
\text { Rif resistant: } \\
17(18.08 \%)
\end{array}
\end{aligned}
$$


Chart.2 Results obtained category wise

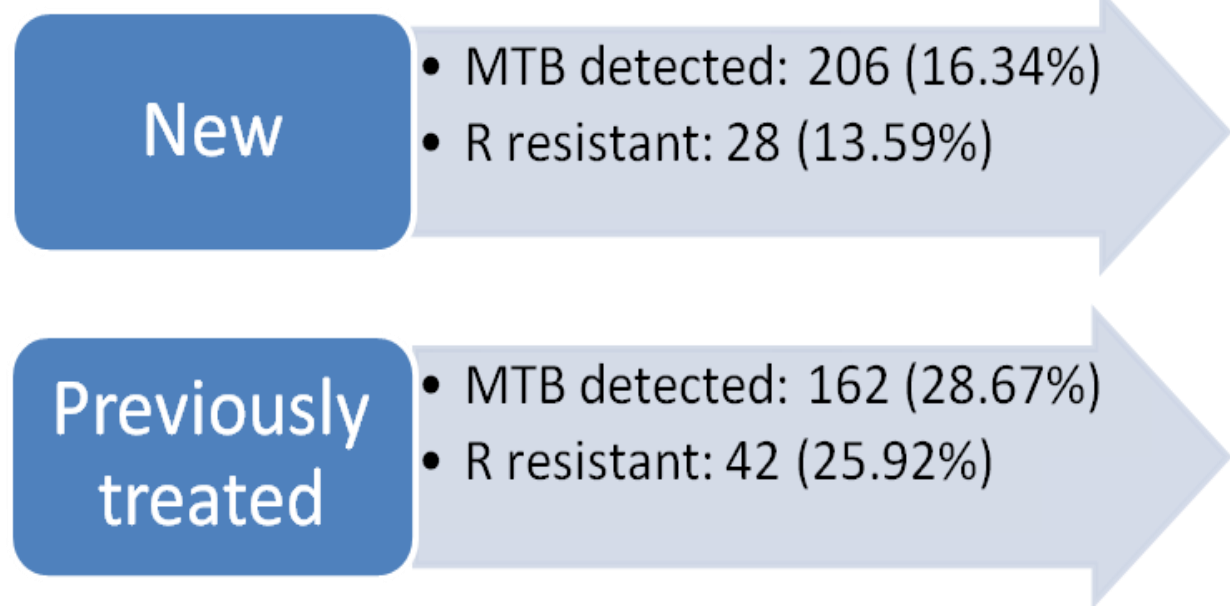

The performance of Truenat has been evaluated extensively by various researchers and has been compared with conventional culture based as well as with other molecular diagnostic methods. Nikam et al., from Hinduja Hospital and Medical research centre, Mumbai evaluated the performance of Truenat RTPCR in comparison with GeneXpert on sputum samples from Pulmonary TB cases and found a high concordance(96\%) with GeneXpert(9).

In another study by Nikam et al., Truenat MTB test was found to have sensitivity of 91.1\% and in- house nested PCR a sensitivity of $90.5 \%(11)$. Though culture based methods are considered gold standard in the diagnosis of tuberculosis, molecular diagnostic methods have been used extensively in the diagnosis of tuberculosis because of the rapidity of the detection of cases which helps in early diagnosis and initiation of treatment. GeneXpert developed by Cepheid company, has long been considered one of the best molecular method because of the ease of doing as it is a cartridge based method which involves simple procedure. But the cost of the instrument and cost per test has been a major drawback for its extensive utilisation in peripheral setups.
Truenat PCR system is a cartridge based test for the rapid diagnosis of tuberculosis along with the detection of rifampicin resistance which is developed in India. With the extensive availability of this economically viable diagnostic method in resource poor country like India will not only help in the early diagnosis but also in the appropriate management and infection control measures and controls the spread of this deadly disease. Truenat will improve linkage-to-care and increase life expectancy also $(2,12)$.

Truenat MTB test is extremely useful test in resource limited health care settings such as designated microscopy centers (DMCs) and primary healthcare facilities in India. It is a rapid test, allows detection of $\mathrm{TB}$ in approximately one hour and can be utilized in near-care settings to provide quick and accurate diagnosis. As a portable platform it could also be utilized in ACF (Active case finding) programs, which are currently the need of the hour.

\section{References}

World Health Organization. Global tuberculosis report 2017 [Internet]. Geneva, Switzerland: World Health 
Organization; 2017. Available from: http://www.who.int/tb/publication s/global_report/en/

Central TB Division. TB India 2017: Revised National Tuberculosis Control Programme: annual status report [Internet]. New Delhi, India: Directorate General of Health Services, Ministry of Health and Family Welfare; 2017. Available

from: https://tbcindia.gov.in/index1.php? lang $=1 \&$ level $=2 \&$ sublinkid $=4728 \& l i d=$ 3275.

Miller, M.B., E.B.Popowitch, M.G.Backlund, et al.,Performance of Xpert MTB/RIF RUO assay and IS6110 real time PCR for Mycobacterium tuberculosis detection in clinical samples, J. Cli. Microbio. 49 (10) (2011) 3458-3462.

Brisson-Noel A, Gicquel B, Lecossier D, LevyFrebault V, Nassif X, et al., (1989) Rapid diagnosis of tuberculosis by amplification of mycobacterial DNA in clinical specimens. Lancet ii: 1069-71.

Beige J, Lokies J, Schaberg T, Finckh U, Fischer M, et al., (1995) Clinical evaluation of a Mycobacterium tuberculosis PCR assay. J Clin Microbiol; 33:90-5.

Golub, J.E., C.I. Mohan et al., Active case finding of Tuberculosis: historical perspective and future prospects, Int.J.Tuberc Lung dis. 9(11)(2005): 1183-1203.

Denkinger CM, Kik SV, Cirillo DM, Casenghi $\mathrm{M}$, Shinnick $\mathrm{T}$, Weyer $\mathrm{K}$, et al., Defining the needs for next generation assays for tuberculosis. J Infect Dis. 2015; 211: S29-S38. pmid:25765104.

Boehme, C.C., P. Nabeta et al., Rapid molecular detection of tuberculosis and Rifampicin resistance, N Eng J Med363 (11)(2010)1005-1015.

Nikam C, Jagannath M, Narayanan MM, Ramanabhiraman V, Kazi M, Shetty A, et al., Rapid diagnosis of Mycobacterium tuberculosis with Truenat MTB: a near-care approach. PLoS One. 2013;8: e51121. pmid:23349670.

Drain PK, Hyle EP, Noubary F, Freedberg KA, Wilson D, Bishai WR, et al., Diagnostic point-of-care tests in resource-limited settings. Lancet Infect Dis. 2014; 14: 239-249. pmid: 24332389.

Nikam C, Kazi M, Nair C, Jaggannath M, M M, $\mathrm{R} \mathrm{V}$, et al., Evaluation of the Indian TrueNAT micro RT-PCR device with GeneXpert for case detection of pulmonary tuberculosis. Int $\mathbf{J}$ Mycobacteriology. 2014;3: 205-210. pmid: 26786489.

David Lee, Nagalingeswara kumaraswamy, Stephen.C, et al., Rapid, point-of-care diagnosis of tuberculosis with novel Truenat assay: Cost-effectiveness analysis for India's public sector PLOS ONE, 2019

Subbaraman R, Nathavitharana RR, Satyanarayana S, Pai M, Thomas BE, Chadha VK, et al., The tuberculosis cascade of care in India's public sector: a systematic review and meta-analysis. PLoS Med. 2016; 13: e1002149. pmid:27780217.

Chadha VK, Anjinappa SM, Dave P, Rade K, Baskaran D, Narang $\mathrm{P}$, et al., Subnational TB prevalence surveys in India, 2006-2012: Results of uniformly conducted data analysis. PLoS One 2019; 14: e0212264.

\section{How to cite this article:}

Vijayalakshmi, J., A. Surekha, A. Renuka devi and Uma Devi, S. 2019. Truenat - A Novel Diagnostic Tool for Rapid Detection of Mycobacterium Tuberculosis and Rifampicin Resistance in Pulmonary Samples. Int.J.Curr.Microbiol.App.Sci. 8(10): 1260-1267. doi: https://doi.org/10.20546/ijcmas.2019.810.148 